\title{
Spiral waves in a surface reaction: Model calculations
}

\author{
M. Bär, N. Gottschalk, M. Eiswirth, and G. Ertl \\ Fritz-Haber-Institut der Max-Planck-Gesellschaft, Faradayweg 4-6, D-14195 Berlin, Germany
}

(Received 20 September 1993; accepted 8 October 1993)

\begin{abstract}
A systematic study of spiral waves in a realistic reaction-diffusion model describing the isothermal CO oxidation on $\mathrm{Pt}(110)$ is carried out. Spirals exist under oscillatory, excitable, and bistable (doubly metastable) conditions. In the excitable region, two separate meandering transitions occur, both when the time scales become strongly different and when they become comparable. By the assumption of surface defects of the order of $10 \mu \mathrm{m}$, to which the spirals can be pinned, the continuous distribution of wavelengths observed experimentally can be explained. An external periodic perturbation generally causes a meandering motion of a free spiral, while a straight drift results, if the period of the perturbation divided by the rotation period is a natural number.
\end{abstract}

\section{INTRODUCTION}

The formation of spiral waves under nonequilibrium conditions has been observed in many chemical and biological systems. ${ }^{1-4}$ Fundamental investigations of their behavior have been carried out extensively with the Belousov-Zhabotinsky (BZ) reaction. ${ }^{5-8}$ Recently, the development of photoemission electron microscopy (PEEM) has also made possible the detailed obscrvation of spatial structures on catalyst surfaces. PEEM reaches a lateral resolution of $\sim 0.2 \mu \mathrm{m}$ in real time, which is necessary because the patterns in question are much finer than, e.g., in the BZ system.

Spirals were found in the $\mathrm{CO}$ oxidation on $\mathrm{Pt}(110)^{9}$ as well as in the reactions of $\mathrm{NO} / \mathrm{NH}_{3}$ and $\mathrm{NO} / \mathrm{CO}$ on $\mathrm{Pt}(100) \cdot{ }^{10,11}$ More quantitative investigations were carried out only with the former system. ${ }^{12} \mathrm{~A}$ couple of interesting phenomena like multiplicity of the spiral period under identical external conditions and resonance phenomena under periodic modulation of a control parameter have been discovered therein. The aim of this paper is the application of a realistic reaction-diffusion model to these effects. The required model was originally derived to explain the temporal (e.g., oscillatory) behavior of the $\mathrm{CO}$ oxidation on $\mathrm{Pt}(110) .{ }^{13}$ Inclusion of spatial coupling via surface diffusion has been used in the study of reaction fronts, solitary waves, and periodic wave trains. ${ }^{14-16} \mathrm{~A}$ variety of unexpected interactions on collision of these waves was successfully modeled by the assumption of local surface defects of mesoscopic size $(5-10 \mu \mathrm{m}) .{ }^{17}$ In addition, we have shown in earlier work that our model can be simplified for the sake of analytical estimates and efficient numerical simulation of two-dimensional wave properties of the medium. ${ }^{18}$ Here we extend this work by giving a systematic discussion of the relation between the physical parameters (the partial pressures and surface temperature) and the parameters of the simplified model. In Sec. III we treat the existence of spirals under excitable, bistable (more precisely doubly metastable), and oscillatory conditions, and estimate the surface defect sizes from the spectrum of observed rotation periods. In Sec. IV the influence of periodic modulation of a control parameter on the movement of a spiral is described.

\section{THE MODEL}

The CO oxidation on $\operatorname{Pt}(110)$ proceeds via the classical Langmuir-Hinshelwood mechanism, ${ }^{19}$ whereby the CO-driven structural change of the $\mathrm{Pt}(110)$ surface needs to be taken into account:

$$
\begin{aligned}
& \mathrm{CO}_{\mathrm{g}}+* \leftrightarrow \mathrm{CO}_{\mathrm{ad}}, \\
& \mathrm{O}_{2 g}+* \rightarrow 2 \mathrm{O}_{\mathrm{ad}}, \\
& \mathrm{O}_{\mathrm{ad}}+\mathrm{CO}_{\mathrm{ad}} \rightarrow 2 *+\mathrm{CO}_{2 g}, \\
& \quad \mathrm{CO}_{\mathrm{ad}} \\
& 1 \times 2 \leftrightarrow 1 \times 1 .
\end{aligned}
$$

(Here * denotes an empty adsorption site, suffices $g$ and ad distinguish gaseous and adsorbed species.) The $\mathrm{CO}$ adsorption follows precursor kinetics. ${ }^{20}$ Moreover, the model should include the asymmetric inhibition, i.e., oxygen adsorption is forbidden on $\mathrm{CO}$ covered sites, but not vice versa.

The sticking coefficient of oxygen depends on the surface structure. Thus three variables-the $\mathrm{CO}$ and $\mathrm{O}$ coverages $u$ and $v$, and the fraction of the surface area existing as a $1 \times 1$ phase $w$-remain, leading to the kinetic equations $^{13}$

$$
\begin{aligned}
\dot{u}= & k_{u} s_{u} p_{\mathrm{CO}}\left[1-\left(u / u_{s}\right)^{3}\right]-k_{2} u-k_{3} u v+\mathrm{D}^{2} u \\
\dot{v}= & k_{v} p_{\mathrm{O}_{2}}\left[s_{v 1} w+s_{v 2}(1-w)\right]\left[1-\left(u / u_{s}\right)-\left(v / v_{s}\right)\right]^{2} \\
& -k_{3} u v \\
\dot{w}= & k_{5}[f(u)-w] .
\end{aligned}
$$

The external control parameters here are the partial pressures of $\mathrm{CO}$ and oxygen, $p_{\mathrm{CO}}$ and $p_{\mathrm{O}_{2}}$, and the crystal temperature $T$, which enters into the rates $k_{2}, k_{3}$, and $k_{5}$ through a Arrhenius relation.

Spatial coupling is introduced through CO diffusion, compared to which $O$ diffusion is very slow. ${ }^{21,22}$ Diffusion 


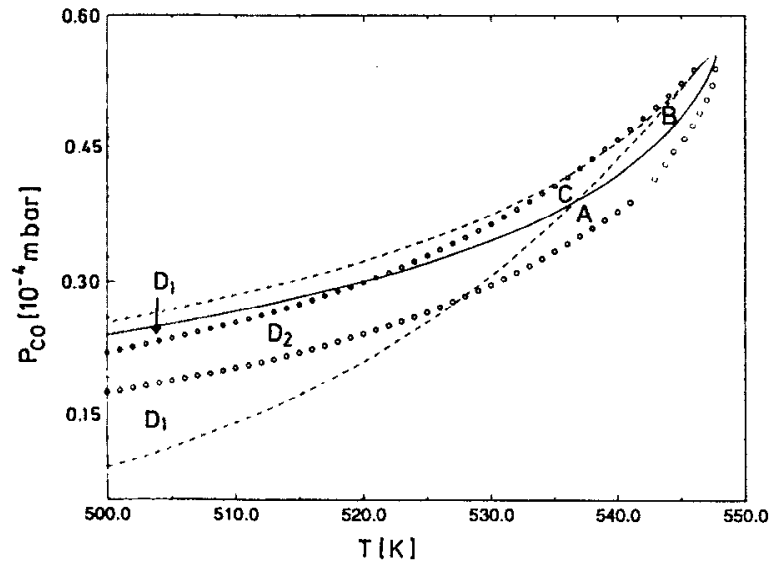

FIG. 1. Phase diagram revealing regions of different dynamical behavior in Eqs. (1) at $p_{\mathrm{O}_{2}}=1.33 \times 10^{-4}$ mbar: (A) and (C) have excitable kinetics (i.e., pulses propagate in the $k_{5} \rightarrow 0$ limit), (B) represents oscillatory kinetics and $\left(D_{i}\right)$ bistability with one $\left(D_{1}\right)$ or two $\left(D_{2}\right)$ metastable states (states unstable against front propagation). Bifurcation of the reaction part are also displayed: Hopf (solid line) and saddle node (dashed line).

on the $\mathrm{Pt}(110)$ surface is anisotropic, i.e., $\mathrm{D}$ is composed of the diffusion constants different in the [1 $1 \overline{1} 0]$ and [001] crystallographic orientations:

$$
\mathrm{D} \nabla^{2} u=D_{x} \frac{\partial^{2} u}{\partial x^{2}}+D_{y} \frac{\partial^{2} u}{\partial y^{2}}
$$

For a detailed discussion of Eqs. (1a)-(1d) the reader is referred to earlier work. ${ }^{13,17}$

A simplified bifurcation diagram of the reaction part of this model is reproduced in Fig. 1, corresponding nullclines are shown in Fig. 2. There are an oscillatory (B), two excitable ( $A$ and $C$ ), and a bistable region (D). In the latter regime it is appropriate to distinguish subregions of qualitatively different behavior. Near the saddle-node and Hopf bifurcations there are areas where only one front solution is possible (labeled $D_{1}$ ), while further inside two different fronts coexist $\left(D_{2}\right)$. We refer to this behavior as "double metastability," since both (stable) fixed points are unstable with respect to a supercritical nucleus.

As long as the two fronts exhibit significantly different velocities, they annihilate when the faster overruns the slower one [Fig. 3(a)]. For comparable velocities, however (i.e., around the middle of region $\mathrm{D}_{2}$ ), they form a bound state [reaction-diffusion pulse, Fig. 3(b)]. This behavior is analogous to results in the excitable domain, where pulses exist only above a certain minimal velocity especially at (not too) high excitation thresholds. ${ }^{16}$ Here the pulse velocity is always determined by the slower one of the two front solutions, consequently the parameter value with equal front velocities is the most favorable for pulse formation. For the $p_{\mathrm{O}_{2}}$ and $T$ values used in Fig. 3 pulses existed between $\mathrm{CO}$ pressures of $2.62 \times 10^{-5}$ and 2.95 $\times 10^{-5}$ mbar (the front velocities were equal at 2.75 $\times 10^{-5}$ mbar).

As shown in previous work ${ }^{15,16} u$ and $v$ are always strictly anticorrelated so that one adsorbate variable suf-
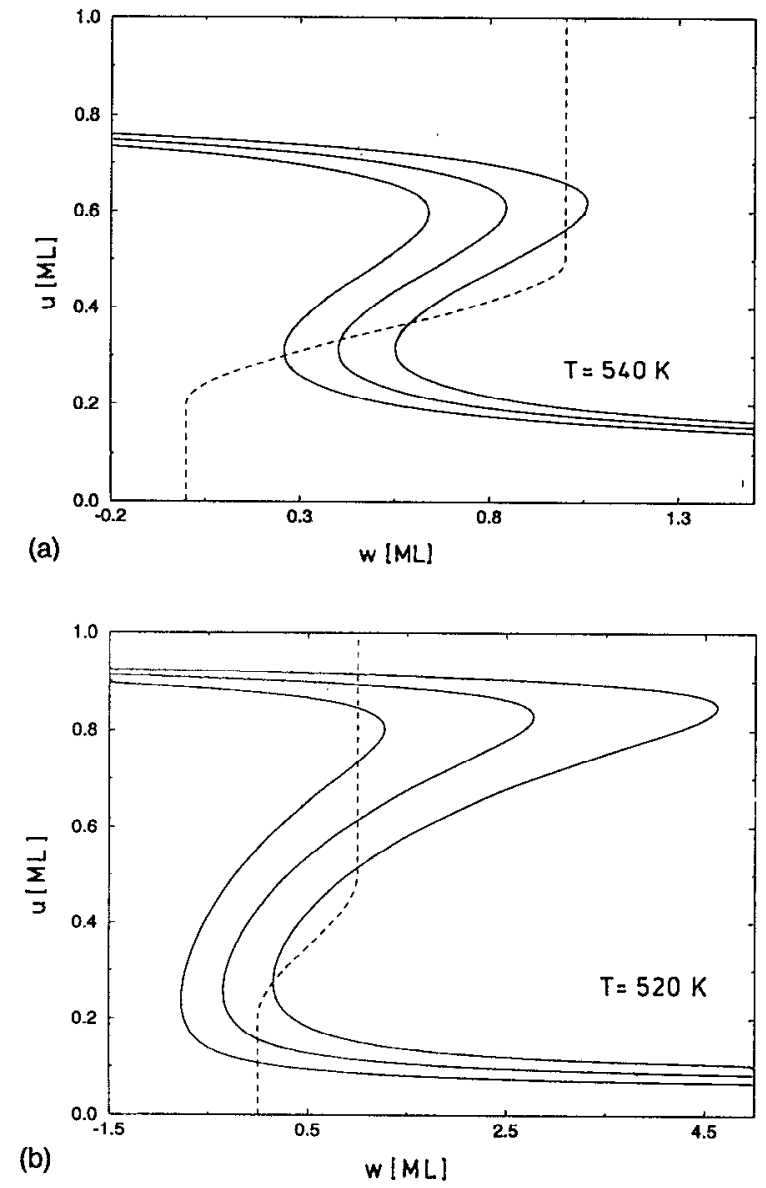

FIG. 2. Nullclines of Eqs. (1) at $p_{\mathrm{O}_{2}}=1.33 \times 10^{-4}$ mbar. The dotted lines represent $d w / d t=0$ and is independent on external parameters. Full lines stand for $d u / d t=0$. (a) $T=540 \mathrm{~K}$. CO pressures from the curve (left to right) are $4.19 \times 10^{-5}$ (excitable, O covered, A), $4.32 \times 10^{-5}$ (oscillatory, B), and $4.45 \times 10^{-5}$ mbar (excitable, $\mathrm{CO}$ covered, C). (b) $T=520 \mathrm{~K}$; all cases lie in the bistable region; $C O$ pressures from left to right are $2.26 \times 10^{-5}$ (stable $O$ covered, metastable CO covered, $D_{1}$ ), $2.66 \times 10^{-5}$ (double metastability, $D_{2}$ ), and $3.06 \times 10^{-5}$ mbar (stable CO covered, metastable $O$ covered state, $D_{1}$ ). Capital letters refer to regions in Fig. 1. A metastable state is unstable against fronts of the inverse covered state.

fices. Hence $v$ is eliminated adiabatically by setting $\dot{v}=0$, solving the resulting algebraic equation for $v=v(u, w)$ and inserting it into Eq. (1a). This leads to a complicated equation for the local change of $u,{ }^{18}$ which for $w$ values in the bistable part of the nulleline can, however, be well approximated with a cubic polynomial (see Fig. 4):

$$
\begin{aligned}
\partial_{t} u= & -k_{0}\left[u-u_{1}(w)\right]\left[u-u_{2}(w)\right]\left[u-u_{3}(w)\right] \\
& +\nabla^{2} u, \\
\partial_{t} w= & k_{5}[f(u)-w] .
\end{aligned}
$$

The new constant $k_{0}$ depends mainly on the temperature and determines the propagation velocity $c$ of fronts and pulses $\left(c \sim \sqrt{D k_{0}}, k_{0} \gg k_{5}\right)$, whereas the rate of structural change $k_{5}$ governs quantities like pulse width or wavelength and period of wave trains in the excitable medium. For further simplification we rescale space and time in order to get dimensionless quantities: 

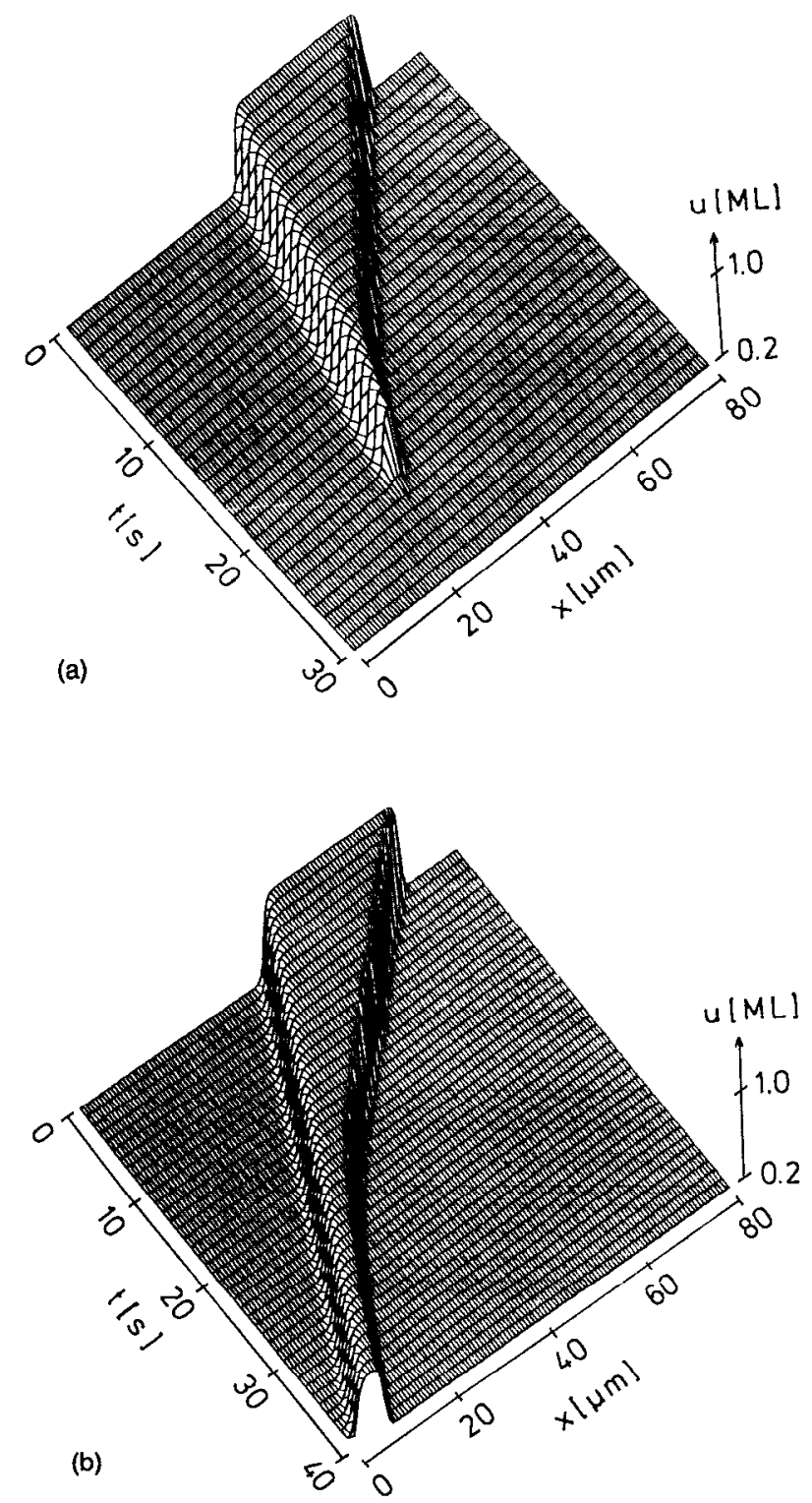

FIG. 3. Interaction of fronts at $T=520 \mathrm{~K}, p_{\mathrm{O}_{2}}=1.33 \times 10^{-4}$ mbar under conditions of double metastability. (a) Annihilation of fronts $\left(p_{\mathrm{CO}}=2.61\right.$ $\left.\times 10^{-5} \mathrm{mbar}\right)$; (b) formation of a stable $\mathrm{CO}$ pulse $\left(p_{\mathrm{CO}}=2.63 \times 10^{-5}\right.$ mbar). The $z$ axis shows the CO coverage $u$.

$$
\hat{x}=\left(\frac{k_{5}}{D_{x}}\right)^{1 / 2} x, \quad \hat{y}=\left(\frac{k_{5}}{D_{y}}\right)^{1 / 2} y, \quad \hat{t}=k_{5} t .
$$

Note that the anisotropy is scaled out now. For efficient numerical integration the $S$-shaped nullcline $u=u(w)$ can be substituted to a good approximation by a $z$-shaped one setting (see Ref. 18)

$$
u_{1}(w)=w_{1}, \quad u_{2}(w)=\frac{w+b}{a}, \quad u_{3}(w)=u_{3} .
$$

Now new variables $\hat{u}$ and $\hat{w}$ are introduced:

$$
\hat{u}=\frac{u_{3}-u}{u_{3}-u_{1}}, \quad \hat{w}=1-w .
$$
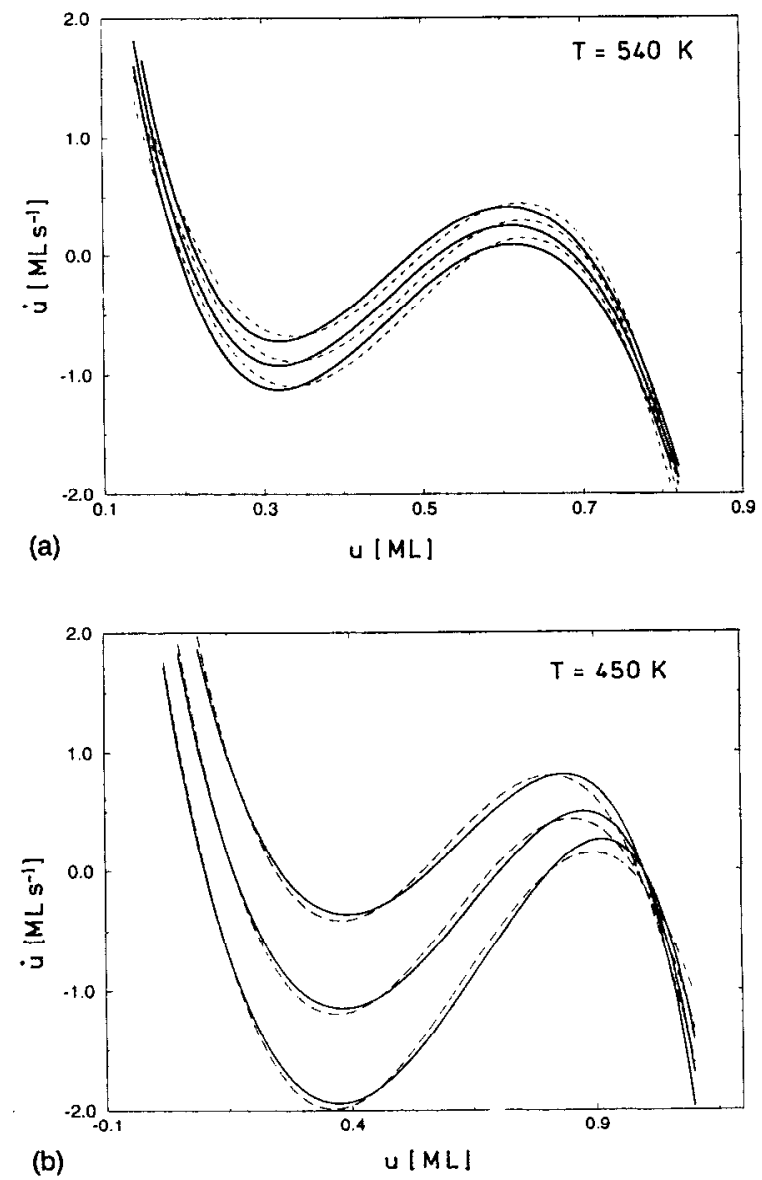

FIG. 4. Plot of the change of the $\mathrm{CO}$ coverage $d u / d t$ against the coverage value $u$ for the $1 \times 1$ surface $(w=1)$ and $p_{\mathrm{O}_{2}}=1.33 \times 10^{-4} \mathrm{mbar}$ (solid lines). (a) $T=540 \mathrm{~K} ; p_{C O}$ from top to bottom: $4.58 \times 10^{-5}, 4.52 \times 10^{-5}$, and $4.46 \times 10^{-5} \mathrm{mbar}$. Fit factors $k_{0}$ for cubic polynomial (dashed lines) were $99.6,98.4$, and $96.5 \mathrm{ML}^{-2} \mathrm{~s}^{-1}$, respectively. (b) $T=450 \mathrm{~K}, p_{\mathrm{CO}}$ from top to bottom: $1.26 \times 10^{-5}, 1.00 \times 10^{-5}$, and $0.74 \times 10^{-5} \mathrm{mbar}$. Fit factors $k_{0}$ for cubic polynomials (dashed line) were $30.5,29.2$, and 28.1 $\mathrm{ML}^{-2} \mathrm{~s}^{-1}$, respectively.

This choice sets the CO-covered stationary state of Eqs. (2) to $(\hat{u}, \hat{w})=(0,0)$, while the oxygen-covered state is represented by $\hat{u}=1$. The variable $\hat{w}$ gives the degree of $1 \times 2$ reconstruction.

The values $u_{1}$ and $u_{3}$ can be obtained from the nullclines. The coverage of the reactive state $u_{1}$ used here is typically 0.2 monolayers (ML). In the bistable regime ( $T$ $<530 \mathrm{~K}$ ) the surface concentration $u_{3}$ of $\mathrm{CO}$ turns out to be $0.95 \mathrm{ML}$. As the temperature is increased, CO desorption sets in and the medium becomes excitable, which is connected with a substantial drop of the $u_{3}$ value for $T>530 \mathrm{~K}$ [cf. $u_{3}=0.65 \mathrm{ML}$ in Fig. 2(a)].

One ends up with the following equations:

$$
\begin{aligned}
& \partial_{t} \hat{u}=-\frac{1}{\epsilon} \hat{u}(\hat{u}-1)\left(\hat{u}-\frac{\hat{w}+b}{a}\right)+\hat{\nabla}^{2} \hat{u}, \\
& \partial_{t} \hat{w}=\hat{f}(\hat{u})-\hat{w},
\end{aligned}
$$

where the abbreviation 


$$
\epsilon=\frac{k_{5}}{k_{0}\left(u_{3}-u_{1}\right)^{2}}
$$

is used. Equations ( $3 a$ ) and ( $3 b$ ) are similar to a model proposed by Barkley ${ }^{23}$ in order to increase computational efficiency. Here, we applied the same algorithm for the integration of the partial differential equations.

The form of $\tilde{f}(\hat{u})$ in Eq. (3b) is simply given by the function $f(u)$ in the new $u$ coordinates and is therefore dependent on the absolute values of $u_{1}$ and $u_{3}$. For excitable conditions $u_{1}=0.2 \mathrm{ML}$ and $u_{3}=0.65 \mathrm{ML}$ have been chosen for all simulations presented here. This leads to

$$
\hat{f}(\hat{u})=\left\{\begin{array}{cc}
0 & \hat{u} \leqslant 1 / 3 \\
1-6.75 \hat{u}(\hat{u}-1)^{2} & 1 / 3<\hat{u}<1 . \\
1 & \hat{u} \geqslant 1
\end{array}\right.
$$

This choice of $\hat{f}(\hat{u})$ ("delayed inhibitor production" ${ }^{24}$ ) stems from fitting the experimental observed ${ }^{25}$ nullcline $\hat{w}(\hat{u})$, and constitutes the crucial difference as compared to standard models. ${ }^{23,26}$

The physical parameter set $\left(p_{\mathrm{CO}}, p_{\mathrm{O}_{2}}, T\right)$ has been mapped into the model parameters $(a, b, \epsilon)$. As examples we show the $T$ dependence of $k_{0}$ as well as the dependence of $b$ on $\Delta T$ and $\Delta p_{\text {Co }}$ (defined as the distance from the oscillatory instability) in Figs. 5(a) and 5(b), respectively. The temperature dependence of $k_{0}$ shows an Arrheniustype form with an activation energy of $6.6 \mathrm{kcal} \mathrm{mol}^{-1}$. Experimental determination of the effective activation energy of front velocities give $20 \mathrm{kcal} \mathrm{mol}^{-1} .{ }^{12}$ Assuming that the diffusion constant has an activation energy of 10 kcal mol ${ }^{-1}, 22$ it was concluded that $k_{0}$ should also exhibit a value of $10 \mathrm{kcal} \mathrm{mol}^{-1}$. However, recent measurements of the $\mathrm{CO}$ diffusion constant under the reaction condition yielded $14 \mathrm{kcal} \mathrm{mol}^{-1}$ (Ref. 27) for the activation energy, implying an activation energy of $6 \mathrm{kcal} \mathrm{mol}^{-1}$ for $k_{0}$, which comes close to the value derived from the model. At this point of the discussion it is important to note that the activation energy of the rate $k_{5}$ of the surface phase transition has an activation energy of $7 \mathrm{kcal} \mathrm{mol}^{-1}$. Therefore the parameter $\epsilon$ should vary only weakly with temperature.

Another quantitative test of the model has been carried out as follows: It is possible to define a center value $\bar{p}_{\mathrm{CO}}$ of the unstable region for constant $p_{\mathrm{O}_{2}}$ and $T$ by taking the value of $p_{C O}$ that yields equal velocities for $\mathrm{CO}$ and $\mathrm{O}$ fronts. For constant $p_{\mathrm{O}_{2}}, \bar{p}_{\mathrm{CO}}$ exhibits an Arrhenius-type $T$ dependence with an activation energy of $6.6 \mathrm{kcal} \mathrm{mol}^{-1}$ in the model and about $5 \mathrm{kcal} \mathrm{mol}^{-1}$ in experiment (cf. Ref. $12, \bar{p}_{\mathrm{CO}}$ is determined as the lowest $\mathrm{CO}$ pressure, where spiral waves occur ${ }^{27}$ ). The ratio $\bar{p}_{\mathrm{CO}} / p_{\mathrm{O}_{2}}$ in the model (e.g., 0.08 at $450 \mathrm{~K}$ ) compares well with the experimental data (0.09 at $450 \mathrm{~K})$.

A discrepancy between model and experiments is given by the fact that the absolute values of the pressures for excitable and oscillatory behavior are considerably higher in experiment. It must, however, be pointed out that in the earlier experimental determination of the rate constants, ${ }^{13}$ the pressures were underestimated by about a factor of 3 (using an ionization gauge) compared to the newer PEEM
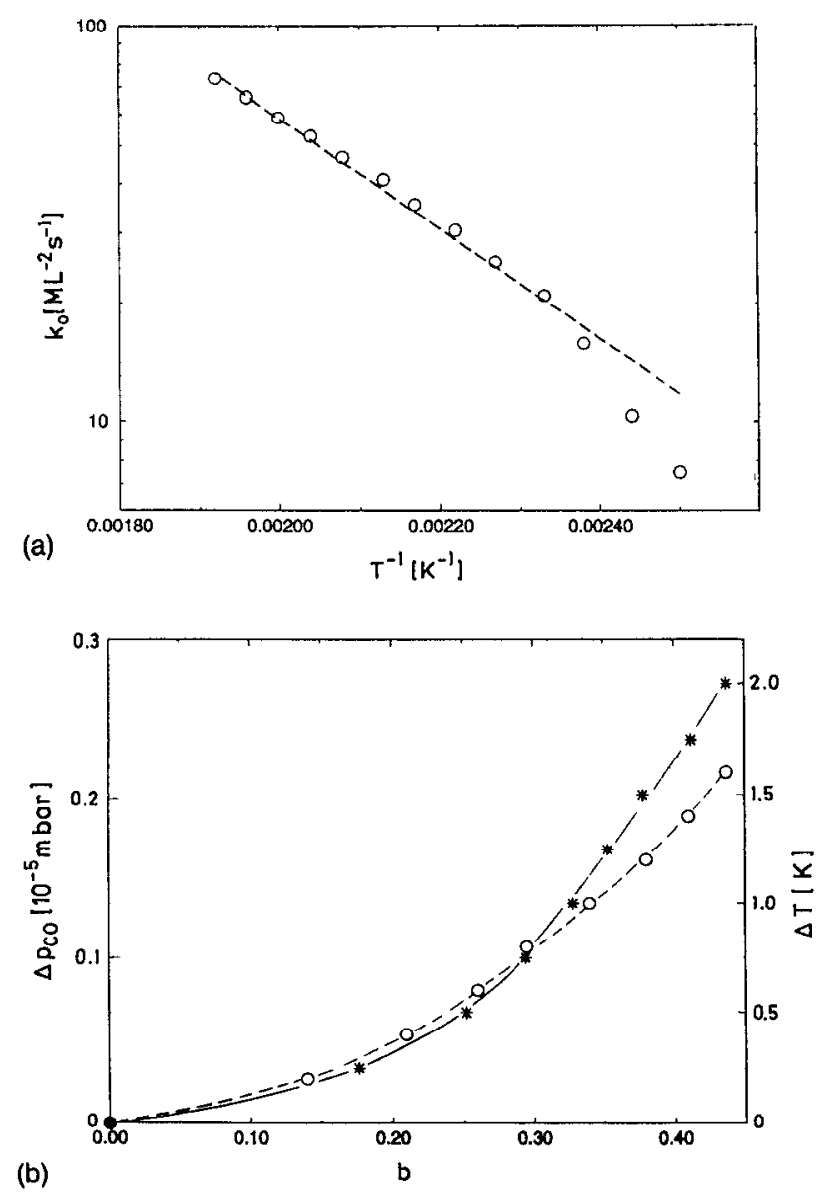

FIG. 5. (a) Dependence of the effective Langmuir-Hinshelwood rate $k_{0}$ on the temperature $T$ for $p_{\mathrm{O}_{2}}=1.33 \times 10^{-4} \mathrm{mbar}$. The fits were carried out near the saddle-node bifurcation at high $p_{\mathrm{CO}}$ ( $\mathrm{cf}$. Fig. 1). The dashed line represent an Arrhenius fit with a activation energy of $6.6 \mathrm{kcal} \mathrm{mol}^{-1}$. (b) Variation of the parameter $b$ of model 3 with the distance from the onset of oscillations at $p_{\mathrm{CO}}=4.42 \times 10^{-5} \mathrm{mbar}, p_{\mathrm{O}_{2}}=1.33 \times 10^{-4} \mathrm{mbar}$, and $T=540 \mathrm{~K}$ given in $\Delta T$ at constant $p_{\mathrm{CO}}$ (stars) and in $\Delta p_{\mathrm{CO}}$ at constant $T$ (circles).

experiments, where the pressures were measured with a baratron. ${ }^{12}$ While this does not influence the ratios of pressures, one has to correct $k_{0}$ when quantitative comparison of absolute values (e.g., periods) between model and experiment are made. Another problem is that the temperature dependence and especially the preexponential factor of $k_{5}$ are difficult to estimate from experiment. ${ }^{25,28,29}$ Moreover, these measurements provide some evidence that the phase transition may proceed differently depending on temperature (displacement of individual $\mathrm{Pt}$ atoms at low, whole parts of rows at high $T$ ). As suggested recently, ${ }^{17}$ the preexponential factor of $k_{5}$ should be chosen about one order of magnitude larger than in the original publication of the model. ${ }^{13}$ Still the rate of the phase transition (which is quite likely also sensitively coverage-dependent) remains to some extent adjustable.

The bifurcations of the reaction part of Eqs. (3) can be constructed from analysis of the corresponding nullclines. Assuming $w_{-}=f(0)=0$ and $w_{+}=f(1)=1$ results in the diagram shown in Fig. 6, very similar to the one of Fig. 1. 


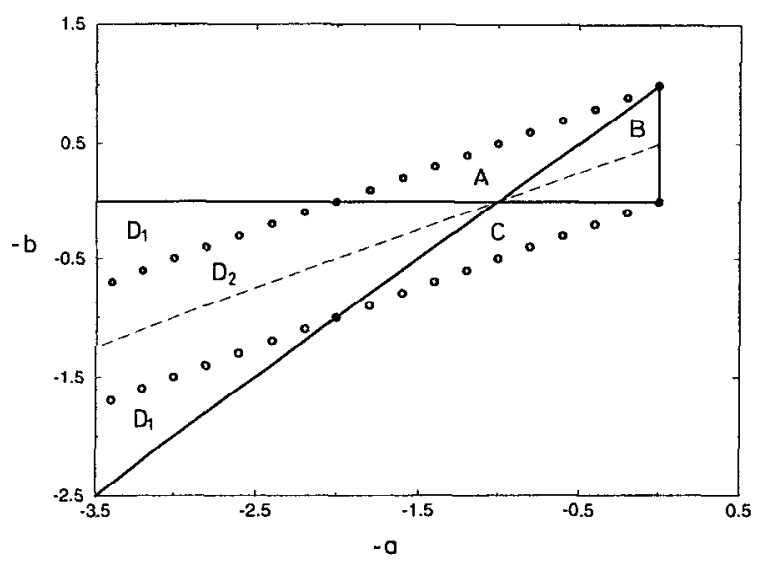

FIG. 6. Phase diagram of the simplified equations (3) corresponding to Fig. 1. The abbreviations and legends are identical to Fig. 1. The boundary of the excitable and the double metastable region (open circles) are calculated in the $\epsilon \rightarrow 0$ limit.

The dispersion relation of plane-wave trains for Eqs. (3) can in good approximation be solved for analytically (cf. Ref. 18). [From now on we drop the ${ }^{\wedge}$ for the quantities of Eqs. (3).] The front velocity for fixed $w_{0}$ is given by

$$
c\left(w_{0}\right)=\sqrt{\frac{1}{2 \epsilon}}\left(1-\frac{2\left(w_{0}+b\right)}{a}\right) .
$$

Triggering a front from a state $w=w_{0}$ will lead to a wave-back at $w=a-2 b-w_{0}$ in the regions where pulses exist. The excitation time $\tau_{\text {exc }}$ and refractory time $\tau_{\text {refr }}$ are

$$
\begin{aligned}
\tau_{\text {exc }} & =\int_{w_{0}}^{a-2 b-w_{0}} \frac{d w}{w_{+}-w}=\ln \left(\frac{w_{+}-w_{0}}{w_{+}-a+2 b+w_{0}}\right), \\
\tau_{\text {refr }} & =\int_{a-2 b-w_{0}}^{w_{0}} \frac{d w}{w_{-}-w}=\ln \left(\frac{a-2 b-w_{0}-w_{-}}{w_{0}-w_{-}}\right) .
\end{aligned}
$$

Taking the finite interface widths $l$ of the pulses into account results in a correction time $\tau_{\text {corr }}$ :

$$
\tau_{\text {corr }}\left(w_{0}\right)=\frac{2 l}{c\left(w_{0}\right)}=\frac{16 \epsilon}{1-2\left(w_{0}+b\right) / a} .
$$

The period $\tau_{\text {per }}\left(w_{0}\right)$ is now given by

$$
\begin{aligned}
\tau_{\text {per }}\left(w_{0}\right)= & \tau_{\text {exc }}\left(w_{0}\right)+\tau_{\text {refr }}\left(w_{0}\right)+\tau_{\text {corr }}\left(w_{0}\right), \\
\tau_{\text {per }}\left(w_{0}\right)= & \ln \left[\frac{\left(w_{+}-w_{0}\right)\left(a-2 b-w_{0}-w_{-}\right)}{\left(w_{0}-w_{-}\right)\left(w_{+}-a+2 b+w_{0}\right)}\right] \\
& +\frac{16 \epsilon}{1-2\left(w_{0}+b\right) / a}
\end{aligned}
$$

which together with Eq. (4) results in the dispersion relation. These values only describe the branch of stable wave train solutions. Note that $\tau_{\text {per }}\left(w_{0}\right)$ diverges for $w_{0}=0$, which means that the system needs an infinity time to relax to the steady state, as well as for $w_{0}=a / 2-b$, where the correction term $\tau_{\text {corr }}$ goes to infinity. Between these ex-

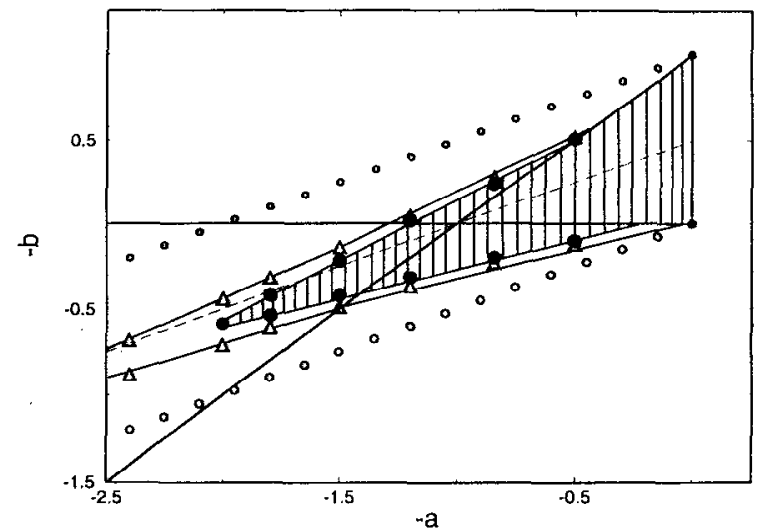

FIG. 7. Existence region of waves in Eqs. (3) for $\epsilon=0.025$. Solid, thick lines, and open circles as in Figs. 1 and 6. Triangles show boundaries of pulse propagation (cf. Fig. 3). Within the hatched region marked by full circles, spiral waves exist.

treme values the function $\tau_{\text {per }}\left(w_{0}\right)$ has a minimum value at $w_{0}=w_{\min }$, which is in the limit of very small $\epsilon$ given by

$$
\tau_{\min }=\tau_{\text {per }}\left(w_{\min }\right)=16\left[\frac{a}{(a-2 b)(2-a+2 b)}\right]^{1 / 2} \cdot \epsilon^{1 / 2},
$$

where $w_{-}$resp. $w_{+}$are set to 0 resp. 1 . This minimum value of $\tau_{\text {per }}\left(w_{0}\right)$ is often identified with the minimum period of a stable wave train in an excitable medium. ${ }^{30}$ The characteristic square root dependence on $\epsilon$ of this minimum period was also obtained by calculating the intersection between the stable and unstable branches of wave train solutions $^{31}$ in a piecewise linearized model.

\section{AUTONOMOUS SPIRAL WAVES}

\section{A. General properties}

We now describe the existence regions and properties of spirals in the parameter space $(a, b, \epsilon)$. In the simulations spirals were obtained using cross-gradient initial conditions in $u$ and $w,{ }^{32}$ e.g., linear profiles between 0 and 1 along the $x$ axis for $u$ and between 0 and $a-2 b$ for $w$ along the $y$ axis were given at the start of the simulation. In reality spirals are created by the break of a plane wave. Then the open ends of the wave usually start to curl and end up in a rotating spiral. The cross-gradients were chosen such that the spiral center was roughly in the middle of the box. The box size was 50 space units realized with 128 gridpoints. For the integration we used the semi-implicit method proposed by Barkley. ${ }^{23}$ Tests with finer grid sizes and time stepping were carried out giving essentially the same results, though a minor shift for the existence region and the properties of spirals was observed.

Figure 7 shows the existence regions of plane waves and spirals (shaded area). While spirals can be obtained everywhere in the oscillatory regime, their existence region under excitable conditions is narrower than that of plane waves-an effect which is even more pronounced in the region of double metastability. Here spirals exist only up to 


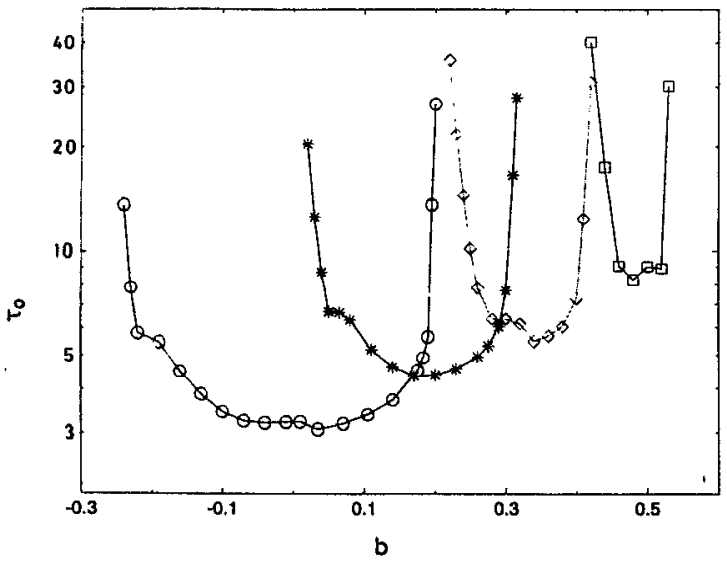

FIG. 8. Rotation periods of spirals in model (3) at $\epsilon=0.025$ for different values of $a$ [from left to right: $a=0.84$ (circles), $a=1.2$ (stars); $a=1.5$ (diamonds) and $a=1.8$ (squares)].

a maximum value of $a$, which depends on $\epsilon$, i.e., for smaller $\epsilon$ the angle between the boundary lines becomes smaller and the regions continue to larger $a$. A criterion for the existence of spiral waves, which was derived recently in the limit of small $\epsilon,{ }^{33}$ can easily be applied to model 3 and reads

$$
a^{2 / 3}<\frac{1}{2}\left(\frac{0.535}{2 \epsilon}\right)^{1 / 2}
$$

For the specific values used in Fig. 7 the limiting value should be $a=2.09$, which is in reasonable agreement with the numerical results $(a=2.00)$. Note that all lines in Fig. 7 run smoothly from one region to another; also no differences in the behavior of the pulses or spirals in the excitable and bistable regime were obtained. The dependence of the periods of spiral rotation $\tau_{0}$ on $b$ for several values of $a$ is reproduced in Fig. 8.

Generally $\tau_{0}$ diverges at the boundaries of the existence region. With increasing $a$, the curves not only become narrower, but are also considerably shifted to higher periods, before diverging off to infinity for the critical value of $a$, above which spirals cease to form.

For fixed $a$, spirals have been studied in dependence on $b$ and $\epsilon$ (Fig. 9). For small $b$, a scaling of the rotation period with $\epsilon^{\alpha}$ was obtained $(\alpha=0.4 \pm 0.05)$ over the entire range, whereas for large $b$ such a scaling was only valid for sufficiently small $\epsilon$. (A scaling law for $\tau_{0}$ has been predicted theoretically ${ }^{34}$ with an exponent $\alpha=1 / 3$.) Going back to physical parameters the scaling becomes

$$
\tau_{0} \propto k_{5}^{\alpha-1} k_{0}^{-\alpha},
$$

i.e., the rotation period scales with the velocity of the phase transition $k_{5}$ and the effective LH rate $k_{0}$. The Arrhenius form of both rate constants leads to a significant decrease of the rotation period for higher temperature. It should be noted here that the range of this scaling property depends on the particular choice of $\hat{f}(\hat{u})$. In the standard case of $\hat{f}(\hat{u})=\hat{u}^{23,26}$ scaling is observed only for $\epsilon<0.01$ (here $\epsilon<0.06)$.

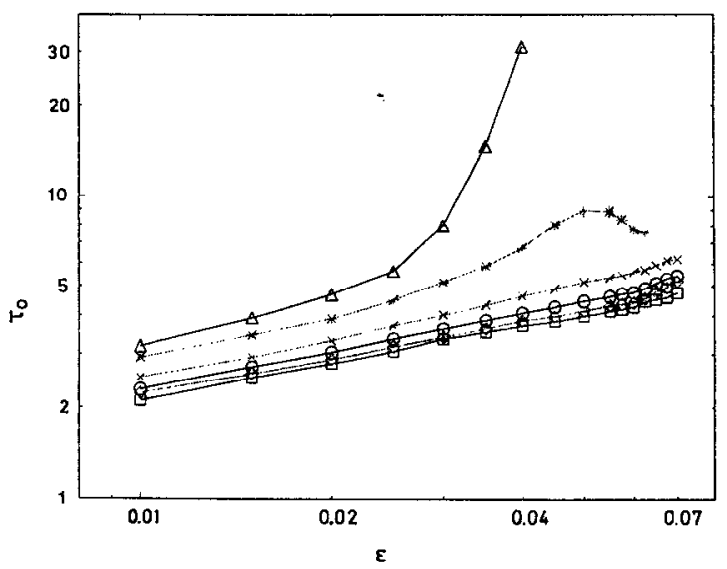

FIG. 9. Scaling behavior of spiral periods against $\epsilon$ for different $b$ at $a=0.84$. The scaling exponent $\alpha\left(\tau_{0} \sim \epsilon^{\alpha}\right)$ is found to be $0.40 \pm 0.02$. The $b$ values are $0.035,0.07,0.105,0.14,0.175$, and 0.19 from bottom to top.

For large $b$ again the period diverged with increasing $\epsilon$, whereas for small $b$ a transition to meandering spirals occurred, as shown in the phase diagram in Fig. 10. Note that the present system contains two regions of meandering: Apart from the standard one towards small $\epsilon,^{26}$ there is another at high $\epsilon$. ( $F$ in Fig. 10 denotes an area of flat, slowly shrinking wave segments.)

Upon further increase of $\epsilon$ at not too high $b$, rigidly rotating $(S)$ or meandering spirals $(M)$ became unstable giving rise to turbulent wave patterns in the region denoted $T$ in Fig. 10. This transition is closely linked to the unusual scaling behavior of the spiral period $\tau_{0}$ (cf. Fig. 9) which leads to an intersection of the spiral period $\tau_{0}$ and the faster growing minimum period $\tau_{\min }$ for wave trains [see Eq.

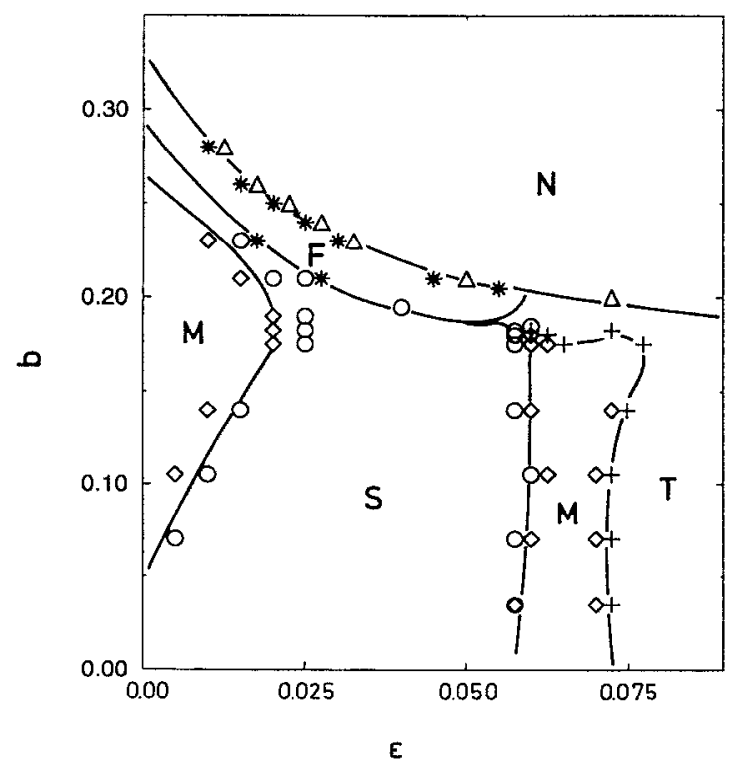

FIG. 10. Phase diagram of different wave types at $a=0.84$ in model (3). $S:$ rigidly rotating spirals; $M$ : meandering spirals; $F$ : flat, shrinking waves; $T$ : turbulence, breakup of spirals; $N:$ no. 
(6)]. The detailed mechanism leading to turbulence has been described elsewhere. ${ }^{24}$

It should be pointed out that the behavior of Fig. 10 with a second meandering and a turbulent region was only obtained for not too high $b$ values in the excitable region $C$ and the oscillatory region B of Fig. 6, but not in the excitable region A nor for conditions of double metastability.

\section{B. Comparison to experiment}

In a recent paper, ${ }^{12}$ Nettesheim et al. gave a systematic account of spiral waves observed in the $\mathrm{CO}$ oxidation on $\mathrm{Pt}(110)$. In particular, double metastability has been shown to support not only coexisting fronts, but also spiral waves. For higher temperature, no evidence for coexisting fronts was obtained anymore, the observed spirals at high $T$ are therefore attributed to excitability.

For fixed control parameters $\left(T=450 \mathrm{~K}, p_{\mathrm{CO}}=4.3\right.$ $\times 10^{-5}$ mbar, $p_{\mathrm{O}_{2}}=4.0 \times 10^{-4}$ mbar) Nettesheim et al. reported a large number of spirals with different rotation periods, ${ }^{12}$ quite unusual for excitable or bistable media. It is, however, well known that spirals can be pinned to artificial nonexcitable cores (e.g., Refs. 35 and 36), which would for the present system correspond to surface defects larger than the core size of free spirals $(\geqslant 1 \mu \mathrm{m}$, dependent on $T$ ).

According to Fig. 5(a) and the required pressure correction (Sec. II) the experimental parameters $(T=450 \mathrm{~K})$ correspond to $k_{0}=90 \mathrm{ML}^{-2} \mathrm{~s}^{-1}$. We study a representative case in the excitable as well as the bistable regime, setting $a$ to 0.84 or 1.8 , respectively. When computing $\epsilon$ according to Eq. (3c), it is necessary to take into account that $u_{1}-u_{3}$ changes from about 0.45 (excitable) to 0.75 (bistable). $b$ is taken near the center of the existence region of spirals for fixed $a$ and $\epsilon$ in the bistable and the COcovered excitable regime, i.e., close to the minimum of $\tau_{0}$ (see Figs. 7 and 8). The saturation of the experimental dispersion curve occurs at a wave velocity $c$ of about 1.6 $\mu \mathrm{m} \mathrm{s}^{-1}$. This corresponds to the velocity of a solitary pulse in the model, from which the diffusion constant $D$ can be calibrated to about $7 \times 10^{-9} \mathrm{~cm}^{2} \mathrm{~s}^{-1}$.

The rate $k_{5}$ of the surface phase transition remains an adjustable parameter, which is used to fit the rotation period of a free spiral. In experiment, most observed periods were around $7 \mathrm{~s}$ (considered to correspond to a free spiral). This results in values for $k_{5}$ of $0.45 \mathrm{~s}^{-1}$ in the excitable and $1.27 \mathrm{~s}^{-1}$ in the bistable region.

The dispersion relations resulting with these parameters are shown in Fig. 11, together with the experimental data points. Note that there is a larger dispersion-free plateau in the bistable than in the excitable case so that no significant dispersion of spirals was observed under bistable conditions. Since spirals of different wavelengths and periods coexist, they compete in such a way that faster spirals will eventually annihilate the slower ones. Figure 12 shows a free spiral (core radius $0.5 \mu \mathrm{m}$ ) and a spiral pinned to a nonexcitable defect of $5 \mu \mathrm{m}$ diameter. The free spiral "wins," while the defect remains as a perturbation in the
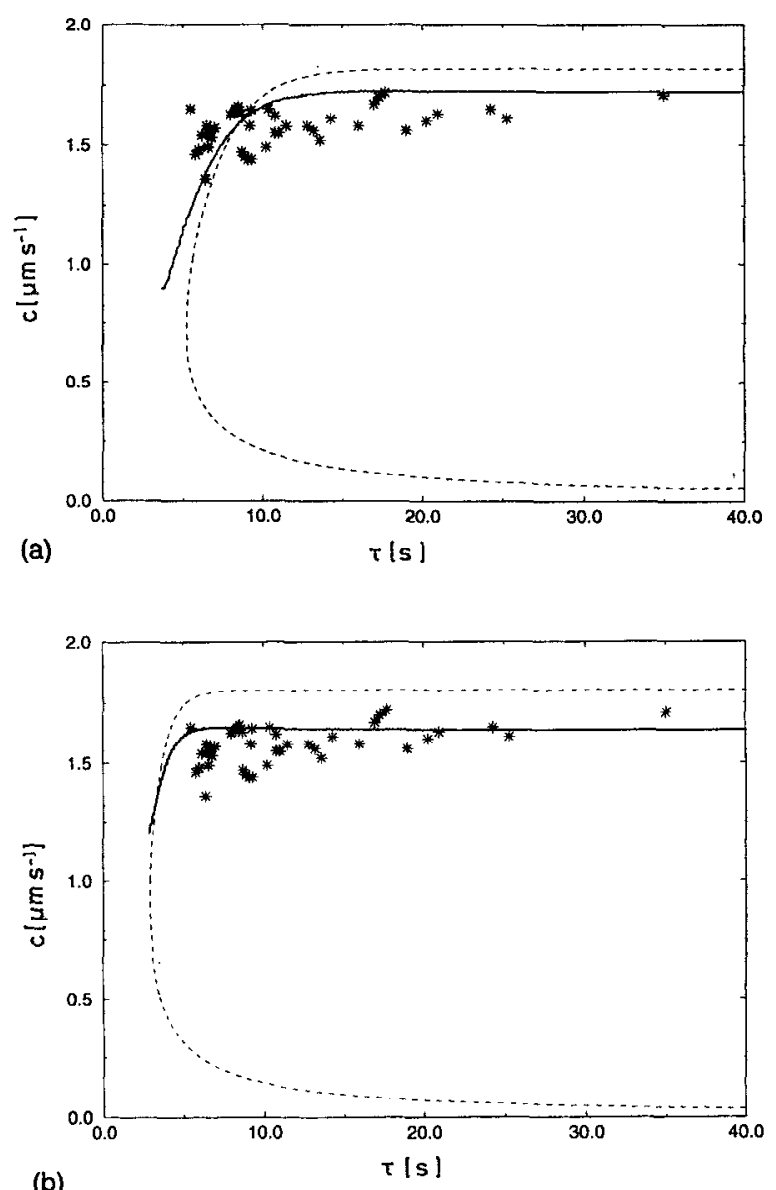

FIG. 11. Dispersion relations, rescaled to physical quantities for (a) excitable parameters ( $a=0.84, b=0.105, \epsilon=0.025$ ) and (b) bistable parameters $(a=1.8, b=0.54$, and $\epsilon=0.025)$. The full lines are obtained by numerical integration of a pulse on a ring in Eqs. (3). Dashed lines show analytical approximation due to Eq. (5), and stars represent experimentally observed spirals.

outer part of the spiral arm. The same behavior has been observed experimentally. ${ }^{12}$

This result directly supports the assumption that the coexistence of different spirals is caused by surface defects. If different free spirals coexisted (due to a multivalued dispersion relation), the core of the extinguished spiral would slowly drift away, as has been confirmed experimentally in the $\mathrm{BZ}$ reaction. ${ }^{37}$

The rotation period $\tau_{\text {rot }}$ depends almost linearly on the defect radius $R_{D}$, as shown in Fig. 13, where the numerical results are compared to the Keener-Tyson formula ${ }^{38}$ (a) and a plane wave propagating along the defect border (b). The largest experimentally observed period ( $35 \mathrm{~s}$ ) corresponds to a defect size of about $15 \mu \mathrm{m}$ while most defects lie below $10 \mu \mathrm{m}$ (cf. Figs. 13 and 14). This is in line with defect sizes estimated from nucleation in the bistable region and the interaction of solitary waves. ${ }^{15,17}$

\section{PERIODICALLY PERTURBED SPIRALS}

Using the kinematic theory of spiral waves ${ }^{39}$ it has been predicted that spirals under the influence of an external periodic perturbation generally exhibit a meandering 


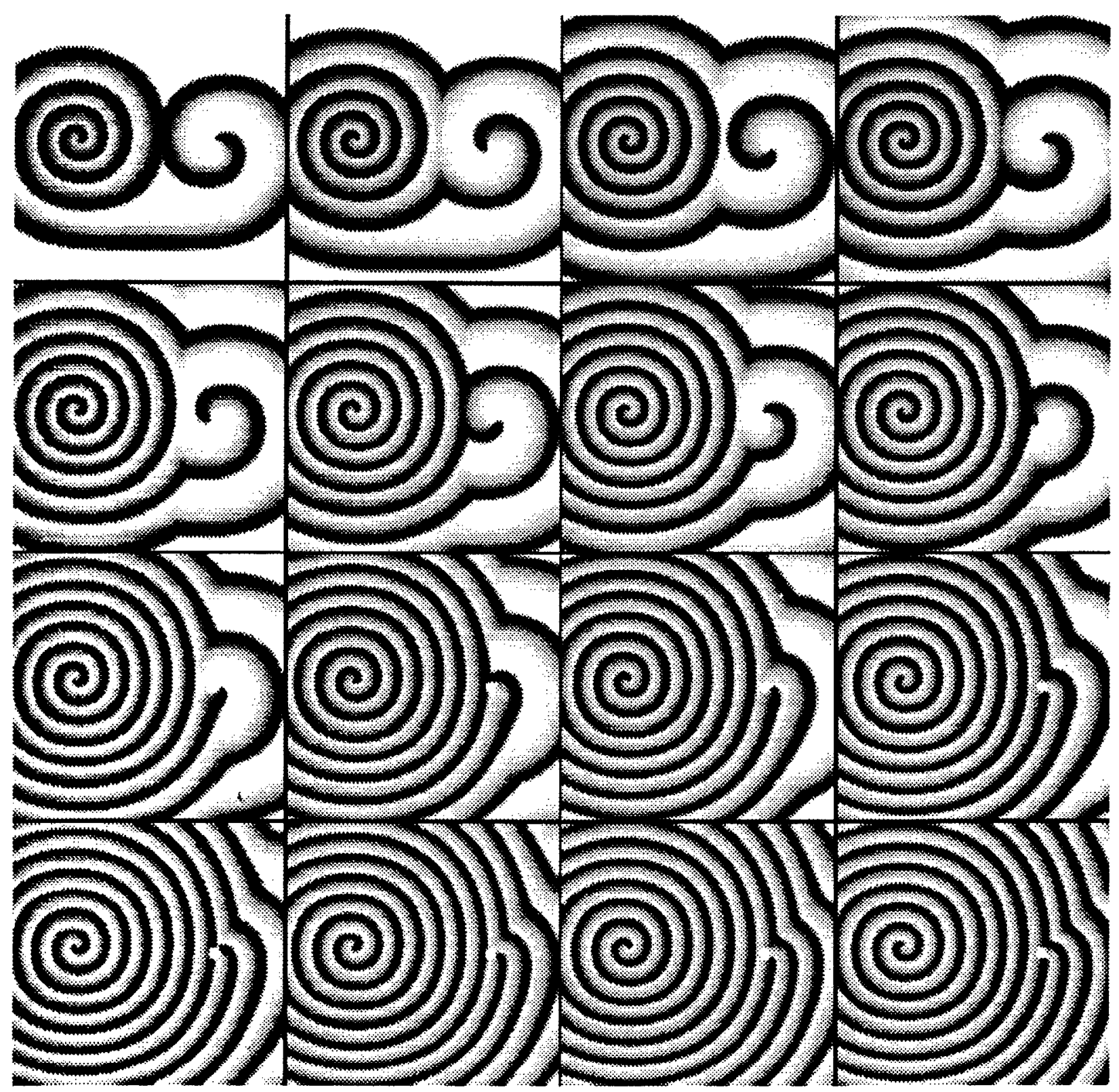

FIG. 12. Competition between a free spiral and a spiral pinned to a defect of $5 \mu \mathrm{m}$ diameter. Picture size is $120 \mu \mathrm{m}$. Time difference between to slides $5.2 \mathrm{~s}$. Model parameters used are $a=0.84, b=0.105, \epsilon=0.025$.

motion, but in the case of resonance (i.e., the perturbation and rotation periods are equal) a linear drift of the core center was predicted and verified experimentally. ${ }^{40} \mathrm{~A}$ natural way to achieve this resonance condition is the use of a spatial gradient of excitability in the medium. Recent experiments with the ruthenium-catalyzed light-sensitive $\mathrm{BZ}$ reaction ${ }^{41,42}$ corroborate these predictions. In this section we first give some general qualitative considerations about spiral resonances, then present simulations using model (3) and compare them to experiments by Nettesheim et al. ${ }^{12}$

\section{A. Qualitative theory of perturbed spirals}

The tip of a rigidly rotating unperturbed spiral performs a circular motion of radius $R_{0}$ with constant period $\tau_{0}$. Under the influence of a sinusoidal modulation of a control parameter $p=p_{0}+\Delta p \cos \omega t$ with $\omega=2 \pi / \tau$ and $\Delta p$ small, the radius $R_{0}$ will vary periodically (for simplicity of the qualitative argument we drop the variation of $\tau_{0}$ for the moment). Consequently the tip trajectory cannot close after $\tau_{0}$, and the center of rotation is shifted, whereby the shift direction depends on the initial phase relationship $\Delta \varphi_{0}$ between spiral rotation and perturbation. In the generic case, the latter is different after every period of the modulation 


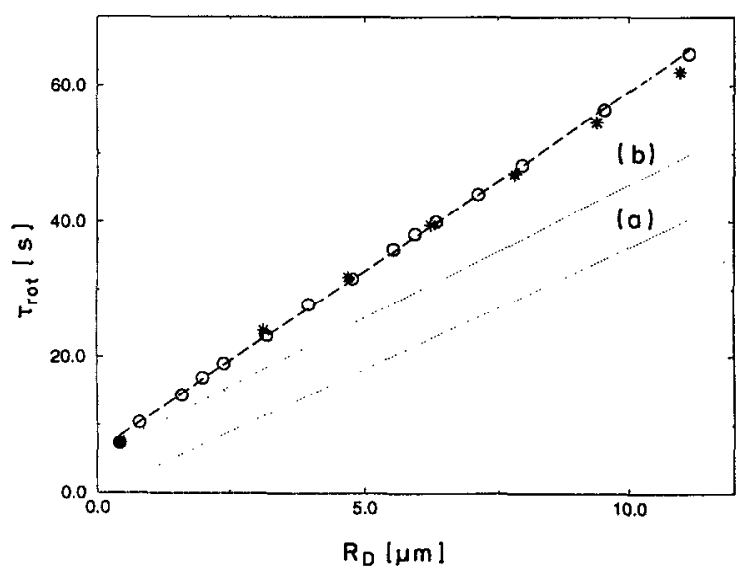

FIG. 13. Rotation period $\tau_{\text {rot }}$ of pinned spirals in dependence on radius $R_{D}$ of the pinning defect. Excitable (circles) and bistable parameters (stars) as in Fig. 11. Dashed lines give analytical estimates due to Keener and Tyson (Ref. 37) (b) and $\tau_{\text {rot }}=2 \pi R_{D} / c_{0}$ (a), which is the KeenerTyson formula in the limit of large $R_{D}$ ( $c_{0}$ is the plane wave velocity; 1.6 $\left.\mu \mathrm{m} \mathrm{s}^{-1}\right)$.

A straight drift results, if and only if $\tau / \tau_{0}=n, n \in N$, i.e., the perturbation period $\tau$ is an integer multiple of the spiral rotation period $\tau_{0}$.

For noninteger ratios

$$
\frac{\tau}{\tau_{0}}=n+\delta ; \quad n \in N, \quad-0.5<\delta<0.5,
$$

the rotation center moves along a circle of radius:

$$
R=w_{r} \Delta r=\frac{\tau_{0}}{\left|n \tau_{0}-\tau\right|} \Delta r
$$

Here the winding number $w_{r}$ denotes the number of perturbation periods per cycle, $\Delta r$ is the shift of the rotation center per $\tau$ (see below). For rational $w_{r}$ (i.e., $\tau / \tau_{0}$ $=n / m ; n, m \in I N, m \neq 1)$ a closed trajectory consisting of $m$ petals is obtained. The sign of $\delta$ determines whether the rotation proper of the spiral and the motion of the rotation center have opposite $(\delta<0)$ or equal $(\delta>0)$ orientation.

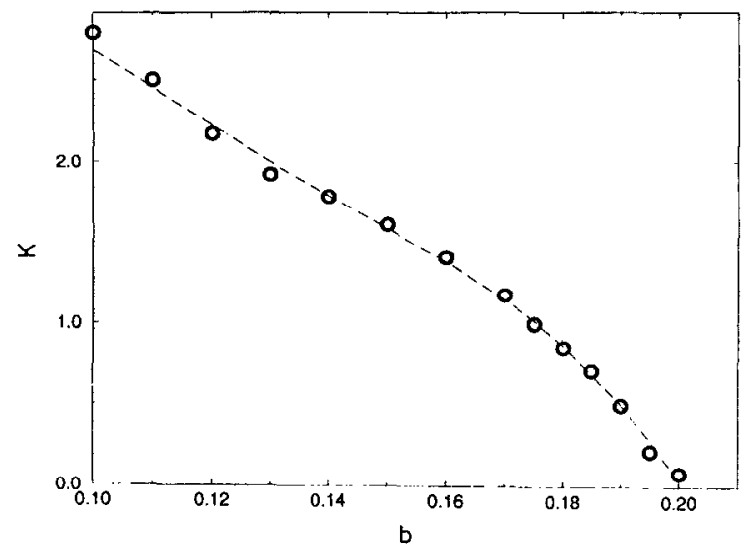

FIG. 14. Curvature of spiral core $\left(K=1 / R_{\text {core }}\right)$ against excitation threshold $b$ in dimensionless units for $a=0.84 . \epsilon=0.025$ in Eqs. (3).
For finite $\Delta p$ the variation of $\tau_{0}$ cannot be neglected. Nevertheless, the above arguments still hold, assuming that $\tau_{0}$ is shifted to a value $\tau_{r}=\tau_{r}(\Delta p)$ and defining $\tau / \tau_{r}$ $=n+\delta$.

In order to estimate the drift velocity we set

$$
\begin{aligned}
& \Delta r_{x(y)}=r_{x(y)}(t+\tau)-r_{x(y)}(t), \\
& \Delta r=\left(\Delta r_{x}^{2}+\Delta r_{y}^{2}\right)^{1 / 2} .
\end{aligned}
$$

The position of the tip at time $t^{*}$ is given by

$$
\begin{aligned}
& r_{x}\left(t^{*}\right)=R_{0}-\int_{0}^{t^{*}} \omega_{0} R(t) \sin \left(\omega_{0} t\right) d t, \\
& r_{y}\left(t^{*}\right)=\int_{0}^{t^{*}} \omega_{0} R(t) \cos \left(\omega_{0} t\right) d t .
\end{aligned}
$$

This ansatz is easily obtained from the integration of the following ordinary differential equation (ODE):

$$
\begin{aligned}
& \dot{r}_{x}=-\omega_{0} R(t) \sin \varphi, \\
& \dot{r}_{y}=\omega_{0} R(t) \cos \varphi, \\
& \dot{\varphi}=\omega_{0}
\end{aligned}
$$

with the initial conditions $r_{x}(0)=R_{0}, r_{y}(0)=0$, and $\varphi(0)=0$. For the autonomous spiral $\left[R(t)=R_{0}\right]$ these equations are a subsystem of the five ODE normal form proposed by Barkley ${ }^{43}$ for the generic behavior of spiral waves. These equations correspond to the case of a steadily rotating spiral. The results sketched here may be generalized to the forcing of meandering spirals by using the full system of five equations. This situation is not relevant for the $\mathrm{CO}$ oxidation treated here, but of interest in the study of forced spirals in the $\mathrm{BZ}$ reaction. ${ }^{44}$

Under the assumption that the curvature $K=1 / R$ of the tip trajectory varies proportionally to the parameter change, i.e.,

$$
R(t)=R_{0} \frac{1}{1-\gamma \cos \omega t}=R_{0}\left(\sum_{n=0}^{\infty} \gamma^{n} \cos ^{n} \omega t\right)
$$

we obtain for $\omega=n \omega_{0}$ (after fixing the phase):

$$
\Delta r_{x}=0 \text { and } \Delta r_{y}=2 \pi n R_{0}\left(\frac{\gamma}{2}\right)^{n} c_{\text {tip }}
$$

( $\gamma$ is the relative amplitude of the curvature in percent). Here, the phase difference between the modulations of parameter and core radius is neglected. Thus a drift occurs only in the $y$ direction. The drift velocity $c_{\text {drift }}$ is given by

$$
c_{\text {drift }}=\frac{\Delta r_{y}}{\tau}=\left(\frac{\gamma}{2}\right)^{n} c_{\text {tip }}
$$

with the normal velocity $c_{\text {tip }}=\omega_{0} R_{0}$ at the tip of the unperturbed spiral.

For a nonharmonic variation of the curvature instead of the $n$th power of $\gamma$ the $n$th Fourier coefficient $a_{n}$ occurs in Eq. ( 8 ) which is given by

$$
R(t)=R_{0}+\sum_{n=0}^{\infty} a_{n} \cos (n \omega t)
$$


The higher resonances $(n>1)$ actually stem from the fact that a sinusoidal perturbation of a parameter causes a variation of the core radius which is not purely sinusoidal, but contains also higher Fourier components. Similar results for drifting spirals as presented here were derived in the framework of the kinematic theory for the cases $n=1$ and $n=2.4,45$

\section{B. Simulations of perturbed spirals}

Drift experiments in the $\mathrm{CO}$ oxidation on $\mathrm{Pt}(110)$ were carried out on spirals with large core (low excitability) and a period of about $66 \mathrm{~s}$ by modulating the temperature by $\pm 1 \mathrm{~K}^{12}$ (Almost) linear drift resulted with a perturbation pcriod of $127 \mathrm{~s}$. The drift velocity (about 0.35 $\mu \mathrm{m} \mathrm{s}^{-1}$ ) was roughly one-third of the plane wave velocity $\left(0.9 \mu \mathrm{m} \mathrm{s}^{-1}\right)$.

For the simulations we chose the parameters given for the excitable case (Sec. III A) with a higher value of $b$ $(0.19)$ to take the lower excitability into account. It should be noted here that although $\epsilon$ was not changed the absolute values of the rates $k_{5}$ and $k_{0}$ are considerably smaller (by a factor of 4) than in Sec. III B, resulting in longer periods due to the rescaling into physical quantities (see Sec. II). This parameter set results in a plane wave velocity of 0.9 $\mu \mathrm{m} \mathrm{s}^{-1}$ in physical units $\left(D=7.0 \times 10^{-9} \mathrm{~cm}^{2} \mathrm{~s}^{-1}\right)$. A variation of the crystal temperature affects all parameters $(a, b, \epsilon)$, but its main effect is a change of the excitability

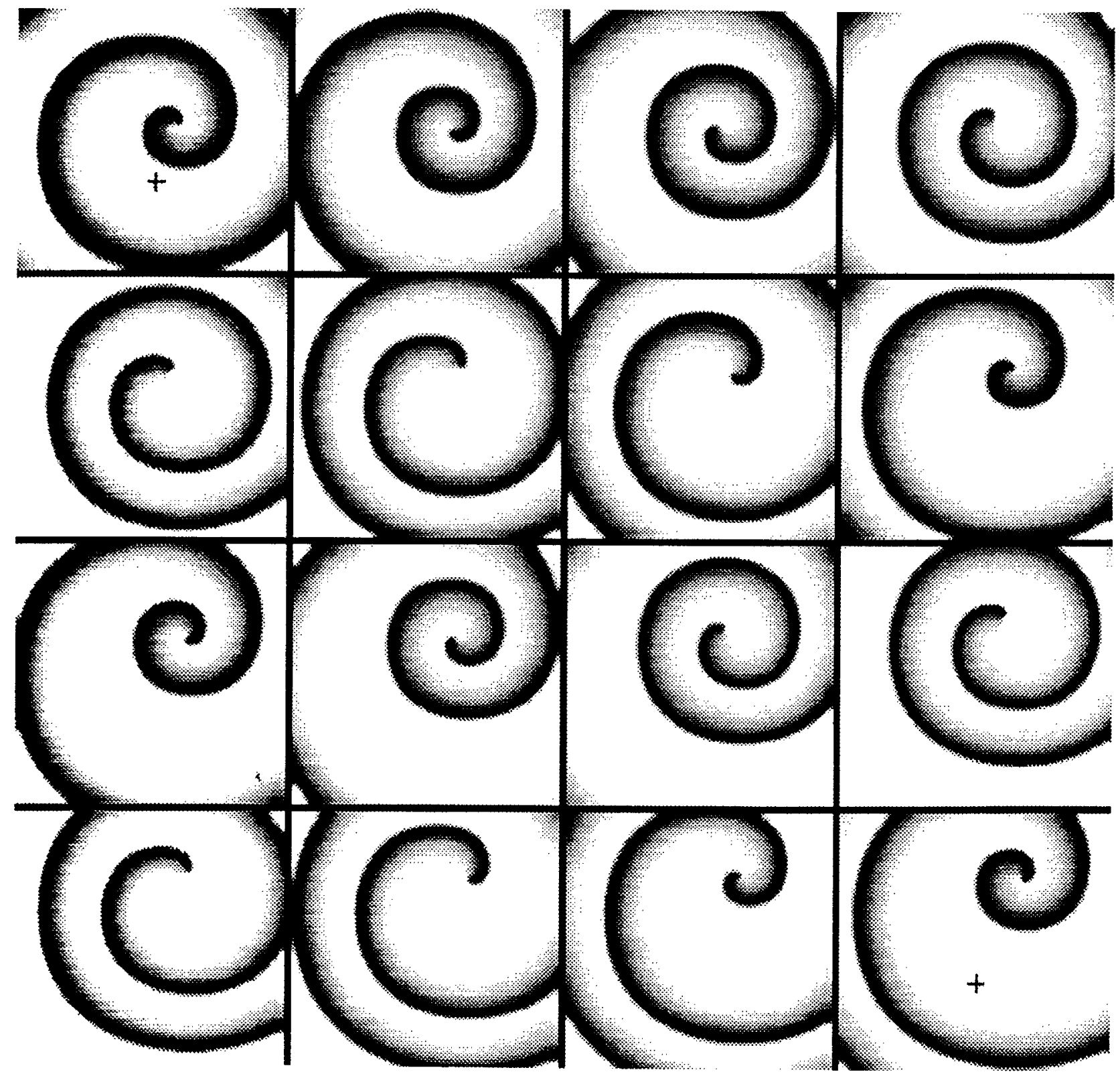

FIG. 15. Dynamics of drifting spiral for ( $a=0.84, b=0.19, \epsilon=0.025)$. Box size $125 \times 125 \mu \mathrm{m}$, time difference $22 \mathrm{~s}$. Spiral period is $70 \mathrm{~s}$, modulation period $155 \mathrm{~s}$. The modulation amplitude is $5 \%$ in $b$. 


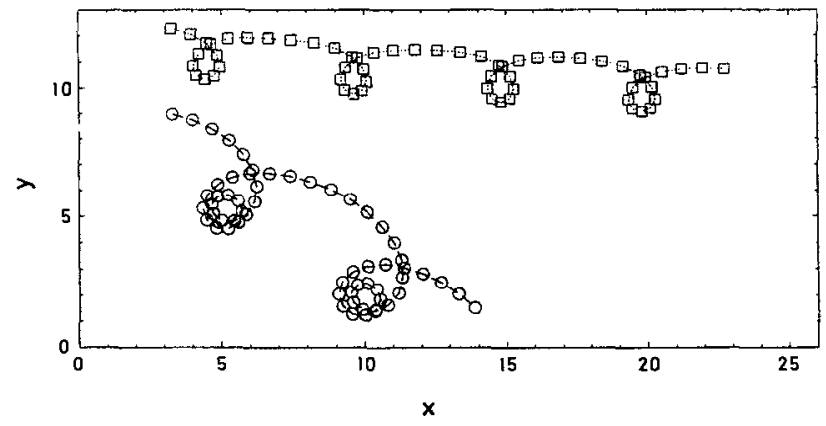

FIG. 16. Comparison of tip trajectories for 1:1 and 1:2 linear drift. The axis shows dimensionless space units $(\mathrm{su})(1 \mathrm{su}=2.5 \mu \mathrm{m})$. Total time in both cases is $330 \mathrm{~s}$. Two points are separated by $5.4 \mathrm{~s}$. For parameters see Fig. 15.

threshold $b$. Under the influence of a $5 \%$ modulation of $b$, the dynamics of the spiral become as shown in Fig. 15. Numerical computation of the tip curvature $K$ vs $b$ revealed a linear relationship to a very good approximation for not too large $\Delta b$, justifying the assumption made in Eq. (7) of Sec. IV A for this particular system.

The spiral tip in the numerical simulations has been defined as the intersection of the $u=1 / 2$ contour with the $w=a / 2-b$ level. The tip trajectories resulting for the different resonances differ characteristically from one another, since $n$ loops are performed before a significant lateral motion along a line of small curvature results (see Fig. 16 for the $1: 1$ and $1: 2$ resonance).

The drift velocity was found to increase linearly and quadratically with the perturbation amplitude $\Delta b$ for small $\Delta b$ in the $1: 1$ and $1: 2$ resonant cases (Fig. 17), respectively. For larger amplitudes the curves flattened. Note that the experimental drift away velocity is reproduced with a large amplitude of $15 \%$.

In Sec. III A the interaction of spirals with surface defects was described. A pinned spiral can also be forced to "take off" and drift with a sufficiently large perturbation.

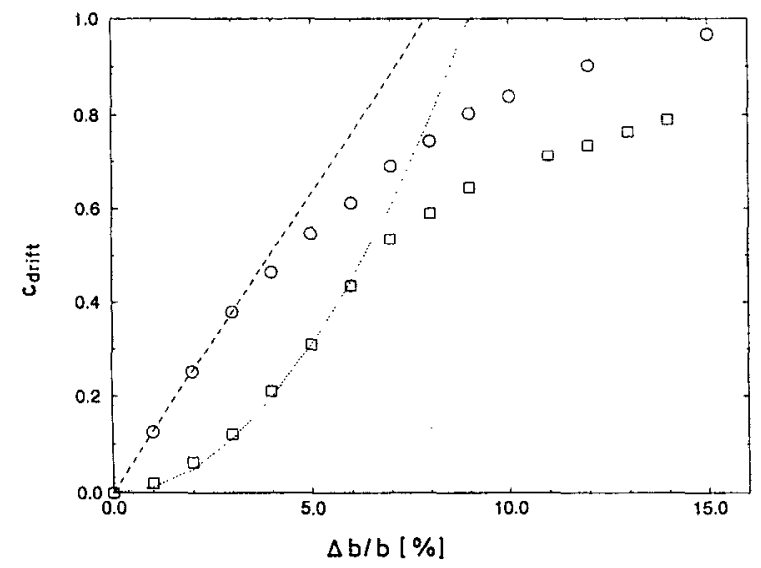

FIG. 17. Drift velocity in dimensionless unit (vu) $\left(1 \mathrm{vu}=0.33 \mu \mathrm{m} \mathrm{s}^{-1}\right)$ dependence on the relative modulation amplitude of $b$ in percent. Parameters as in Fig. 15. (a)
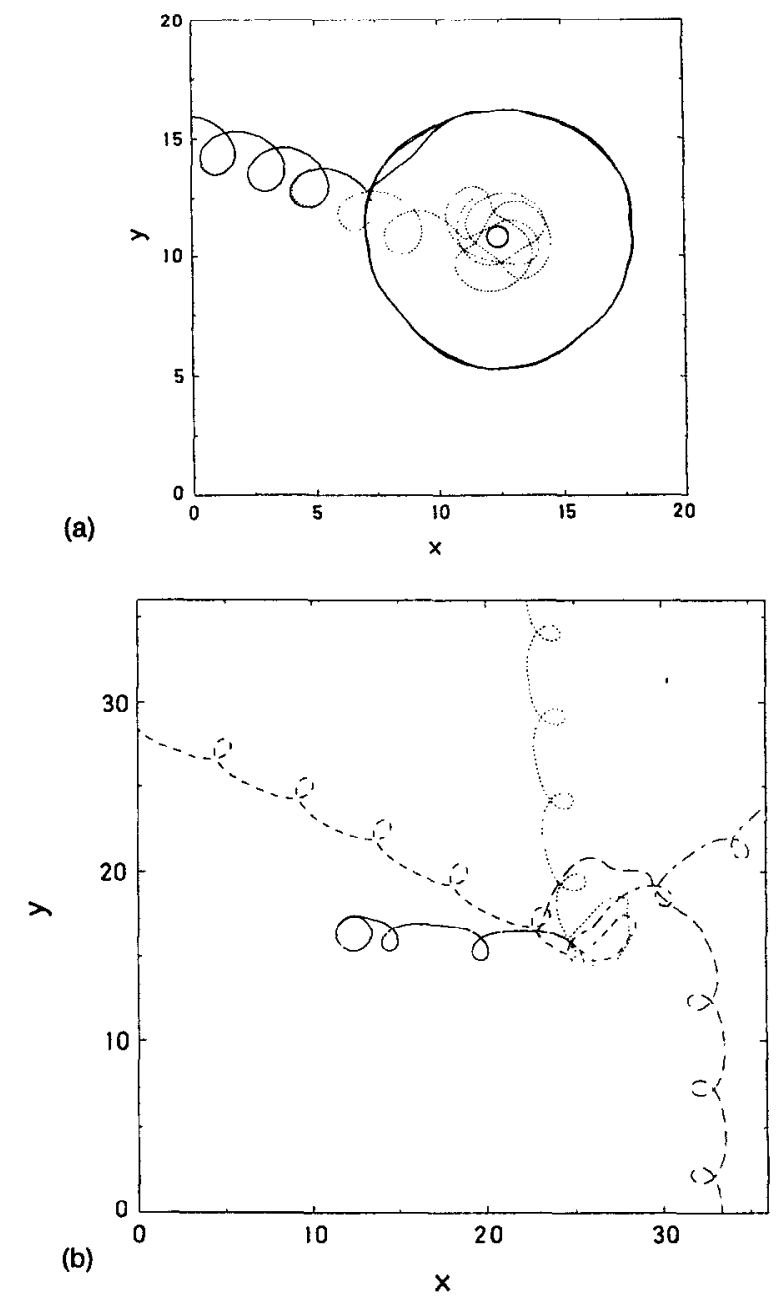

FIG. 18. (a) Pinning of linearly drifting spirals at a small ( $1 \mu \mathrm{m}$ radius, dotted line) and large defect ( $12 \mu \mathrm{m}$ radius); modulation amplitude $3 \%$. Tip trajectories are shown. (b) Scattering of linearly drifting spiral (solid line) at defects of different radii [ $1 \mu \mathrm{m}$ (dashed); $1.7 \mu \mathrm{m}$ (dot dashed); $3.1 \mu \mathrm{m}$ (dotted); $8.7 \mu \mathrm{m}$ (long dashed)]. Spatial axis as in Fig. 16, parameters as in Fig. 15; modulation amplitude 5\%.

On the other hand, a drifting spiral may get pinned when running into a large enough defect [Fig. 18(a)]. If the defect is smaller the tip spends some time in its vicinity, but gets "depinned" by the modulation [Fig. 18(b)]. This leads to a sharp change of the drift direction (depending on the defect size and the initial conditions, i.e., the phase of the spiral). Generally, the transition from pinning to scattering behavior of drifting spirals happens at a critical forcing amplitude for a given defect size (e.g., $5.3 \%$ for a defect radius of $6.2 \mu \mathrm{m}$ ). Similar results were obtained in the study of the interaction between drifting spirals and defects in spatially inhomogeneous media with a gradient of excitability. ${ }^{46}$

\section{DISCUSSION}

The reconstruction model of CO oxidation on $\mathrm{Pt}(110)$ has been simplified to a modified FitzHugh-Nagumo-type system. The crucial difference in standard models of excitable media ${ }^{26}$ is the delayed inhibitor production (cf. Fig. 
2), giving rise to two additional (unstable) fixed points. This does not only lead to significant quantitative differences but also to qualitatively new behavior, namely a second meandering region and turbulence due to spiral breakup. ${ }^{24}$ The delay in the inhibitor production comes into play because the structural phase transition of the $\mathrm{Pt}(110)$ surface does not set in until the $\mathrm{CO}$ coverage reaches a threshold value of $0.2 \mathrm{ML}$. In equilibrium, the $1 \times 1$ vs $\mathrm{CO}$ coverage [i.e., the nullcline $\dot{w}=0$ from Eqs. (1c) and (3b), respectively] has actually been determined directly in experiment, both with low-energy electron diffraction (LEED) and scanning tunnel microscopy (STM). ${ }^{25,28}$ Thus no arbitrary assumption enters the model in this respect.

It was shown that the behavior in the excitable regions stretches continuously into the bistable one (cf. Fig. 6). The latter was subdivided depending on the types of wave solutions, which are in complete qualitative agreement with experiment. ${ }^{12}$ The $\mathrm{CO}$ oxidation on $\mathrm{Pt}(110)$ is to our knowledge the first system in which double metastability (i.e., coexisting fronts, pulses, and spirals under bistable conditions) has been obtained both experimentally and in a realistic model.

Achievement of quantitative agreement between experiment and theory causes some problems. The kinetics of the phase transition is certainly too complicated to be described by a simple Arrhenius term, but not known in sufficient detail from experiment. There is evidence that the microscopic mechanism of the phase transition may change with temperature. In addition, for not too high temperature, further structural changes (facetting) also influencing oxygen adsorption come into play. ${ }^{47,48}$ Satisfying quantitative results have been obtained by treating the rate $k_{5}$ of the phase transition as an adjustable parameter (within certain bounds), the other parameters being known fairly accurately from experiment. ${ }^{13}$

It was demonstrated that the coexistence of many spiral rotation periods for identical external parameters is almost certainly due to pinning of the spiral to artificial cores, i.e., surface defects. Such defects are unavoidable in practice. They presumably consist of small patches which are rough on an atomic scale (e.g., because of an enhanced density of dislocations or steps) or a local accumulation of impurities, leading to kinetic parameters (e.g., sticking coefficients) which differ from the values for a perfect $\mathrm{Pt}$ surface. Adjusting $k_{5}$ such as to match the experimental data, the size distribution of these defects could be estimated (roughly $1-10 \mu \mathrm{m}$, very few larger). One expects that even smaller defects are more likely to occur (as can be verified in STM studies, e.g., Ref. 49). However, defects smaller than the diffusion length (roughly $1 \mu \mathrm{m}$ in the present system) do not have a significant impact on the pattern formation through reaction and diffusion, consequently they do not enter the considerations here.

Under the influence of an external periodic perturbation, rigidly rotating spirals started to meander, both under excitable and doubly metastable conditions. A linear drift (modulated traveling wave ${ }^{43}$ ) resulted if and only if the perturbation period was an integer multiple of the spiral rotation period. The mentioned defects caused scattering or pinning of the drifting spirals, depending on their size.

\section{CONCLUSIONS}

The CO oxidation on $\operatorname{Pt}(110)$ can be described by a simple two-variable model with delayed inhibitor production. The calculations of wave phenomena, in particular autonomous and periodically perturbed spirals, are in good qualitative and even quantitative agreement with experiment, both under conditions of excitability as well as double metastability. Surface defects play an important role for the spatiotemporal self-organization.

\section{ACKNOWLEDGMENT}

The authors are indebted to S. Nettesheim and A. S. Mikhailov for fruitful discussions.

${ }^{1}$ A. T. Winfree, When Time Breaks Down (Princeton University, Princeton, 1987).

${ }^{2}$ Nonlinear Wave Processes in Excitable Media, edited by A. V. Holden, M. Markus, and H. G. Othmer (Plenum, New York, 1991).

${ }^{3}$ Spatio-Temporal Organization in Nonequilibrium Systems, edited by $S$. C. Müller and T. Plesser (Projekt, Dortmund, 1992).

${ }^{4}$ A. S. Mikhailov, Foundations of Synergetics I (Springer, Berlin, 1990).

${ }^{5}$ K. I. Agladze and V. I. Krinsky, Nature 296, 424 (1982).

${ }^{6}$ S. C. Müller, T. Plesser, and B. Hess, Science 230, 661 (1985).

${ }^{7}$ G. S. Skinner and H. L. Swinney, Physica D 48, 1 (1991).

${ }^{8}$ J. Schütze, O. Steinbock, and S. C. Müller, Nature 356, 45 (1992).

${ }^{9}$ S. Jakubith, H. H. Rotermund, W. Engel, A. v. Oertzen, and G. Ertl, Phys. Rev. Lett. 65, 3013 (1990).

${ }^{10} \mathrm{G}$. Veser, F. Esch, and R. Imbihl, Catal. Lett. 13, 371 (1992).

${ }^{11}$ G. Veser, F. Mertens, A. S. Mikhailov, and R. Imbihl, Phys. Rev. Lett. 71, 935 (1993).

${ }^{12}$ S. Nettesheim, A. v. Oertzen, H. H. Rotermund, and G. Ertl, J. Chem. Phys. 98, 9977 (1993).

${ }^{13}$ K. Krischer, M. Eiswirth, and G. Ertl, J. Chem. Phys. 96, 9161 (1992).

${ }^{14}$ M. Bär, M. Falcke, C. Zülicke, H. Engel, M. Eiswirth, and G. Ertl, Surf. Sci. 269/270, 471 (1992).

${ }^{15}$ M. Bär, C. Zülicke, M. Eiswirth, and G. Ertl, J. Chem. Phys. 96, 8595 (1992).

${ }^{16}$ M. Falcke, M. Bär, H. Engel, and M. Eiswirth, J. Chem. Phys. 97, 4555 (1992).

${ }^{17}$ M. Bär, M. Eiswirth, H. H. Rotermund, and G. Ertl, Phys. Rev. Lett. 69, 945 (1992).

${ }^{18}$ M. Bär, M. Falcke, and M. Eiswirth, Physica A 188, 78 (1992).

${ }^{19}$ T. Engel and G. Ertl, Adv. Catal. 28, 1 (1979).

${ }^{20}$ R. P. H. Gasser, An Introduction to Chemisorption and Catalysis by Metals (Clarendon, Oxford, 1985).

${ }^{21}$ R. Gomer, Rep. Prog. Phys. 53, 917 (1990)

${ }^{22}$ A. v. Oertzen, Ph.D. thesis, Freie Universität, Berlin (1992).

${ }^{23}$ D. Barkley, Physica D 49, 61 (1991).

${ }^{24}$ M. Bär and M. Eiswirth, Phys. Rev. E (in press).

${ }^{25}$ T. Gritsch, D. Coulman, R. J. Behm, and G. Ertl, Phys. Rev. Lett. 63, 1086 (1989).

${ }^{26}$ A. T. Winfree, Chaos 1, 303 (1991).

${ }^{27}$ S. Nettesheim, Ph.D. thesis, Freie Universität, Berlin (1993).

${ }^{28} \mathrm{~K}$. Heinz, A. Barthel, L. Hammer, and K. Müller, Surf. Sci. 191, 174 (1987).

${ }^{29}$ T. Gritsch, D. Coulman, R. J. Behm, and G. Ertl, Appl. Phys. A 49, 403 (1989).

${ }^{30}$ J. Rinzel, Biophys. J. 15, 975 (1975).

${ }^{31}$ R. N. Khramov, Biophysics 23, 871 (1978)

${ }^{32}$ W. Jahnke and A. T. Winfree, Int. J. Bifurc. Chaos 1, 445 (1991).

${ }^{33}$ A. Karma, Phys. Rev. Lett. 66, 2273 (1991).

${ }^{34}$ A. Karma, Phys. Rev. Lett. 68, 397 (1992).

${ }^{35}$ A. M. Pertsov, E. A. Ermakova, and A. V. Panfilov, Physica D 14, 117 (1984).

${ }^{36}$ O. Steinbock and S. C. Müller, Phys. Rev. E 47, 1506 (1993).

${ }^{37}$ V. I. Krinsky and K. I. Agladze, Physica D 8, 50 (1983). 
${ }^{38}$ J. J. Tyson and J. P. Keener, Physica D 32, 327 (1988).

${ }^{39}$ V. A. Davydov, V. S. Zykov, and A. S. Mikhailov, Sov. Phys. Usp. 34, 665 (1991).

${ }^{40}$ K. I. Agladze, V. A. Davydov, and A. S. Mikhailov, Sov. Phys.-JETP Lett. 45, 767 (1987).

${ }^{41}$ M. Markus, Z. Nagy-Ungvarai, and B. Hess, Science 257, 225 (1992).

${ }^{42} \mathrm{M}$. Braune and $\mathrm{H}$. Engel, in Spatio-Temporal Organization in Nonequilibrium Systems, edited by S. C. Müller and T. Plesser (Projekt, Dortmund, 1992), p. 34.
${ }^{43} \mathrm{D}$. Barkley (to be published).

${ }^{44} \mathrm{M}$. Braune and H. Engel Chem. Phys. Lett. 211, 534 (1993).

${ }^{45}$ A. S. Mikhailov, V. S. Zykov, and V. A. Davydov, Physica D (submitted).

${ }^{46}$ X. Zou, H. Levine, and D. A. Kessler, Phys. Rev. E 47, R800 (1993).

${ }^{47}$ J. Falta, R. Imbihl, and M. Henzler, Phys. Rev. Lett. 64, 1409 (1990).

${ }^{48} \mathrm{M}$. Sander and R. Imbihl, Surf. Sci. 255, 61 (1991).

${ }^{49}$ T. Gritsch, D. Coulman, R. J. Behm, and G. Ertl, Surf. Sci. 257, 297 (1991). 